\title{
Interference-Aware Channel Assignment in Wireless Mesh Networks
}

\author{
Rosario G. Garroppo, Stefano Giordano, Davide Iacono, Stefano Lucetti, Luca Tavanti \\ Dept. of Information Engineering \\ University of Pisa, Italy \\ \{name.surname\}@iet.unipi.it
}

\begin{abstract}
Keywords - wireless mesh networks, IEEE 802.11s, multichannel, interference modelling, graph theory, radio resource management.
\end{abstract}

\section{EXTENDED ABSTRACT}

The increased popularity and the growth in the number of deployed IEEE 802.11 Access Points (APs) have raised the opportunity to merge together various disjointed wireless LANs to form a unique wireless mesh network (WMN). In this architecture only few nodes, called Portals (MPPs), interface to the external world thanks to their bridging/gateway functions, while several Mesh Points (MPs) form a wireless backbone acting as relay nodes. But MPs are also able to provide network access to user devices (stations) that are inside their coverage range (thus becoming Mesh Access Points, MAPs). As a result, WMNs are supposed to handle a large amount of traffic, including flows that demand specific requirements, such as VoIP calls or multimedia broadcasting.

To this end, one serious limit of 802.11-based WMNs is the fact that the standard plans to use the CSMA/CA access protocol over a single frequency/channel [1]. In the Mesh architecture, where the MPs shall transmit their traffic and at the same time forward what they receive from other MPs, inter-flow and intra-flow interference can easily grow to unbearable levels, causing the network to offer poor performance in terms of throughput, delay, and jitter [2][3].

In this scenario, the use of multi-channel is not an option but an obligation. Many channel assignment algorithms and protocols have already been proposed to solve this problem at physical and/or data link layers. A distributed channel assignment solution has been presented in [5], while a distributed assignment joined with routing has been developed in [7]. A centralized approach was instead the focus of [8] and [9], which exploited graph theory. Ref. [10] and [11] merged routing with centralized channel assignment. The above solutions do not foresee a 802.11 MAC layer modification, whereas other works that utilize channel switching on a single radio do need it [12][13].

Now we propose a channel assignment algorithm for WMNs that accounts for a much more detailed modelling of the interference. Differently from previous works, we consider a physical interference model, developed according to radio propagation rules in order to account for the real signal power received from each interference source. In fact, the common protocol model used in most previous works assumes binary interference: a node is either within or out of the interference range of another. This means that a node that is very close to another, interferes with it in the same manner as a node on the edge of the interference range, which could actually be very large. The path loss experimented by the signal is generally not taken into account. Clearly these assumptions drive to a rough estimate of the interference, while a more realistic computation can provide for a much finer tuning of channel assignment.

Moreover, not only the interference among MPs will be considered, but also the interference that comes from external sources, like other WLANs or devices working in the same frequency band (e.g. radars). Interference that can arise among radios on the same node will be taken into account as well. In fact, due to path loss absence, it can be even more critical than others. Finally, we also include the possibility that a node is equipped with directional antennas (besides the classical omni-directional ones).

Our algorithm is based on the assumption of a treetopology wireless backbone (which is the one usually created in IEEE 802.11s WMNs [4]), where each node can be equipped with several network interfaces. The intended solution will exploit a centralized approach like in [8], where a central server performs the assignment. It works independently from the specific radio technology of a mesh node. In this manner we avoid any MAC modification that would be very troublesome and may lead to interoperability troubles. Moreover, we do not address joint channel assignments and routing issues, since route changes usually occurs on a much shorter time scale than frequency assignments, eventually leading to scalability problems and network instability.

In order to properly account for the interference in the channel assignment problem, the intended approach will be to employ the graph theory [14]. In particular, a conflict graph [15] will be built to consider the various links in the wireless backbone that interfere with each other. While in a connectivity graph we represent each MP with a vertex and each wireless link with an edge, in the conflict graph each vertex represents a wireless link and the vertices are connected only if they interfere with each other. Each edge in the conflict graph has a weight that is proportional to the amount of interfering power received from all sources. Our aim is to assign channels to NICs (Network Interface Card) in order to disconnect the conflict graph vertices as much as possible; ideally the graph will be completely disconnected after the channel allocation.

Our problem can be formalised as follows:

- Let $n=1 \ldots N$ be the MPs (nodes) in the network; 
- Let $G(V, E)$ be the connectivity graph, where $v \in V$ are the nodes (vertices), and $e \in E$ the wireless links (edges);

- Let $G_{c}(L, S)$ be the conflict graph, where $l \in L$ are the wireless links (vertices) and $s \in S$ the edges that indicate interference between them;

- Let $C=\{c \mid c=1,2, \ldots, K\}$ be the complete set of channels and $C_{n}$ the subset of channels available on node $n$ (due to external sources, e.g. radar, some channels may not be usable in some parts of the network);

- Let $\beta\left(c_{l}, c_{g}\right)$ be a factor that accounts for the spectrum overlapping between channels $c_{l}$ and $c_{g}$;

- Let $\rho_{n}$ be the utilization factor of node $n$, which quantifies the fraction of time it is in the transmission state;

- $\quad$ Let $S_{m}{ }^{n}(h)$ be the power received at the $m^{\text {th }}$ interface of node $n$ from the interference source $h$ (including the antenna gain in the direction of $h$ );

- Let $\gamma_{m, p}^{n}\left(c_{m}, c_{p}\right)$ be a factor that measures the interference on the interface $m$ of node $n$ (which works on channel $c_{m}$ ) due to couplings with interface $p$ (on board to the same node) working on channel $c_{p}$.

For each link $l=(i, j)$ between nodes $i$ and $j$, we compute the function $f_{l}$ with the objective of finding the channel $c \in C_{l}=C_{i} \mathrm{n} C_{j}$ that minimizes the interference on link $l$ :

$$
\begin{aligned}
& f_{l}=\min _{C_{l}} \sum_{g} W_{l, g}(c)+\Gamma_{i}(c, m)+\Gamma_{j}(c, q) \\
& W_{l, g}(c)=\beta\left(c, c_{g}\right)\left[\left(1-\rho_{i}\right)\left(\rho_{h} S_{m}^{i}(h)+\rho_{k} S_{m}^{i}(k)\right)+\right. \\
& \left.+\left(1-\rho_{j}\right)\left(\rho_{h} S_{q}^{j}(h)+\rho_{k} S_{q}^{j}(k)\right)\right] \\
& \Gamma_{i}(c, m)=\sum_{p \neq m} \gamma_{m, p}^{i}\left(c_{m}, c_{p}\right)
\end{aligned}
$$

In the formulas, $g$ represents a generic interfering link (between nodes $h$ and $k$ ) that is connected to $l$ in the conflict graph. $W_{l, g}(c)$ is the weight assigned to the edge between $l$ and $g$ and is a function of the signal power $S(\cdot)$ received at nodes $i$ and $j$ from nodes $h$ and $k$ according to physical signal propagation model, in the frequency band of channel $c$. In addition, it also a function of $\Gamma_{i}$ and $\Gamma_{j}$, defined in (3), which compute the sum of all interferences due to other NICs installed on nodes $i$ and $j$. These sources are often very relevant, since, in spite of the best isolation techniques, they do not experiment path loss and may thus generate a signal power comparable to the one received from other nodes. Still in (2), by means of the terms $1-\rho_{i}$ and $1-\rho_{j}$, we also consider the fraction of time the nodes $i$ and $j$ are in receiving state, as this is the time they are subject to destructive interference. Similarly, we also count the utilization factor of $h$ and $k$ ( $\rho_{h}$ and $\rho_{k}$ respectively), which are the fraction of times they may cause interference. The utilization factors allow us to consider the traffic that flows into the network, so that we have a very accurate knowledge of the average channel conditions.

The problem of optimal channel assignment in an arbitrary mesh topology has been proven to be NP-hard based on its mapping to a graph-colouring problem [6]. For this reason, our approach will be heuristic. The proposed algorithm integrates the one proposed in [8] with the Breadth
First Search (BFS) exploration priority used in [9]. In addition to these approaches, we consider, as previously underlined, a physical interference model and the possibility of having directional antennas. The algorithm whose flowchart is shown in Figure 1 is used to compute the function $f_{l}$.

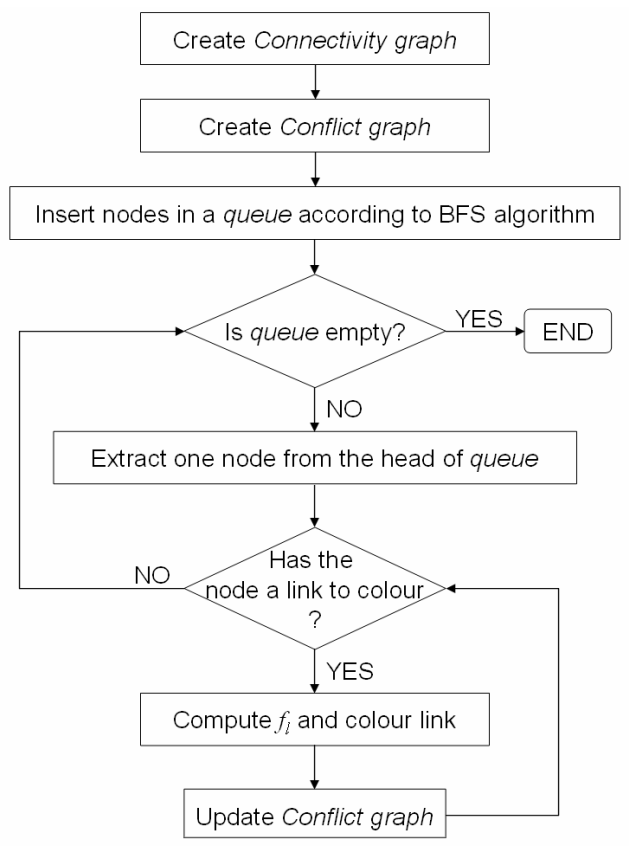

Figure 1. Flowchart for the proposed algorithm

BFS is defined in [14]. We refer to the connectivity graph. Starting from the root, we insert all nodes connected to it (level one) in a list of nodes to be visited (say queue). These nodes may be ordered (the criterion is irrelevant). Then, for each of these add every children node (level two) at the bottom of the list (as long as it has not been added before) and order them. Follow the same procedure (level three, four, etc.) until all the vertices in the tree have been added.

As we can see from Fig.1, the connectivity graph and the conflict graph are built (at the beginning we assume that all interfaces are momentarily tuned on the same channel - the most unfavourable case) and the queue of nodes is filled. Next, the nodes are visited and, when a node has some link to be coloured (i.e. a channel must be assigned to that link), the algorithm computes function $f_{l}$ as defined in (1) in order to minimize the interference for the considered link. The outcome is a channel that is definitively assigned to that link. The conflict graph is then updated, as, following to the allocation, some vertices will presumably be disconnected. In case one node has some links to be coloured but all its radios have been definitively tuned, the algorithm will reuse one of the already set channels. In this context, it is important to account for directional antennas, as some channels may be in use on interfaces that do not guarantee the destination to be in the coverage field. The procedure is repeated until all nodes have been visited and therefore all links have been assigned their final channel.

The performance of our proposed algorithm is evaluated, using a simulative approach, in terms of traffic throughput and end-to-end delay. 


\section{REFERENCES}

[1] IEEE standard 802.11-2007, June 2007.

[2] J.Li, C.Blake, D.De Couto, H.Lee, R.Morris, "Capacity of ad hoc wireless networks", MobiCom, July 2001.

[3] M.Gerla, K.Tang, R.Bagrodia, "TCP performance in wireless multihop networks", Second IEEE Workshop on Mobile Computing Systems and Applications, Feb. 1999.

[4] IEEE Unapproved draft P802.11s/D1.02, March 2007.

[5] B.Ko, V.Misra, J.Padhye and D.Rubenstein, "Distributed Channel Assignment in Multi-Radio 802.11 Mesh Networks", WCNC 2007.

[6] A.Raniwala, Kartik Gopalan, Tzi-Cker Chiueh, "Centralized Channel Assignment and Routing Algorithms for Multi-channel Wireless Mesh Networks", ACM SIGMOBILE Mobile Computing and Communications Review (MC2R), April 2004.

[7] A.Raniwala, Tzi-cker Chiueh, "Architecture and algorithms for an IEEE 802.11-based multi-channel wireless mesh network", INFOCOM, March 2005.

[8] M. K. Marina, S.R.Das, "A topology control approach for utilizing multiple channels in multi-radio wireless mesh networks", $2^{\text {nd }}$ International Conference on Broadband Networks, Oct. 2005.

[9] K. N. Ramachandran, $\quad$ E. M. Belding, $\quad$ K. C. Almeroth, M. M. Buddhikot, "Interference-Aware Channel Assignment in MultiRadio Wireless Mesh Networks", INFOCOM, April 2006.

[10] P. Kyasanur and N.H. Vaidya, "Routing and interface assignment in multichannel multi-interface wireless networks", Wireless Communications and Networking Conference, 2005.

[11] M.Alicherry, R.Bhatia, and L.E.Li. "Joint channel assignment and routing for throughput optimization in multi-radio wireless mesh networks", MobiCom, pages 58--72, 2005.

[12] Paramvir Bahl, Ranveer Chandra, John Dunagan, "SSCH: Slotted Seeded Channel Hopping for Capacity Improvement in IEEE 802.11 Ad-Hoc Wireless Networks", Proceedings of the 10th annual Int. Conf. on Mobile computing and networking, pages 216-230, 2004.

[13] J.So, N.Vaidya, "Multi-channel MAC for ad hoc networks: handling multi-channel hidden terminals using a single transceiver", MOBIHOC, May 2004, pp. 222-233.

[14] K.Rosen, "Discrete Mathematics and its Applications", McGraw Hill, 1999.

[15] Kamal Jain, Jitendra Padhye, Venkat Padmanabhan, Lili Qiu, "Impact of Interference on Multi-hop Wireless Network Performance", Proc. of Int. Conf. on Mobile Computing and Networking, pp. 66-80, San Diego, CA, Sept. 2003. 